\title{
Luciferase gets deep and sensitive
}

A luciferin analog enables highly sensitive bioluminescent imaging from deep within biological tissue samples.

The bioluminescent firefly enzyme luciferase is commonly used as a reporter in biological systems. Light generated by the action of luciferase on its substrate, luciferin, can be used to report gene expression or for various other applications, such as tracking labeled cells in whole animals. However, its use is often restricted to the surface of samples because the wavelength of the light generated is too short to penetrate tissue.

To overcome this limitation, Shinae Kizaka-Kondoh and colleagues at Tokyo Institute of Technology developed a substrate that leads to bioluminescence at a longer wavelength and thus can be detected from deeper within tissues. They previously synthesized one such substrate, known as AkaLumine, but it was not soluble enough for practical use. In their new work, they synthesized the analog AkaLumine- $\mathrm{HCl}$, which is soluble at concentrations of $40 \mathrm{mM}$ or lower.

In contrast to the natural D-luciferin substrate, which produces bioluminesence in the yellow-green range, AkaLumine- $\mathrm{HCl}$ has a benzothiazole moiety in place of the aromatic group and leads to emission in the near-infrared range.

The authors tested the performance of AkaLumine- $\mathrm{HCl}$ in tissues and cancer cell lines and detected at least 6.7-fold more bioluminescence in $8-\mathrm{mm}$ tissue sections than produced with D-luciferin and its analog CycLucl. Moreover, compared with other substrates, AkaLumine-HCl led to maximal emission at lower concentrations-a potential advantage, as limited substrate availability can interfere with quantification across samples.

The authors tested AkaLumine- $\mathrm{HCl}$ in mice by injecting it into subcutaneous tumors. The signals observed were 40 -fold stronger than those generated using D-luciferin, making AkaLumine- $\mathrm{HCl}$ comparable to CycLuc1. It was also used to detect signals emanating from metastases in mouse lung, a tissue that is difficult to image given its position deep within the animal; the signal was more than eightfold stronger than that generated from D-luciferin and threefold stronger than that from CycLuc1.

The combination of sensitivity, red-shifted emission and effectiveness at low concentrations suggests that AkaLumine- $\mathrm{HCl}$ could be particularly useful for imaging tissue deep within animals.

Richard Pattison

\section{RESEARCH PAPERS}

Kuchimaru, T. et al. A luciferin analogue generating near-infrared bioluminescence achieves highly sensitive deep-tissue imaging. Nat. Commun. 7 , 11856 (2016). 